keeping side of the service is towards electronic rather than electro-mechanical equipment; and on the observational side towards the impersonal photographic plate rather than the over-fallible human senses. As always, the attainment of extreme precision is costing much effort ; and if there exists any fleeting regret at the passing of the pendulum, which has served us well for nearly three centuries, it should be allayed by increasing confidence in the last decimal place of the published time-signal corrections.

\section{WAVE GUIDES IN ELECTRICAL COMMUNICATION}

$\mathrm{A}^{\mathrm{T}}$ T a meeting of the Wireless Section of the Institution of Electrical Engineers on November 3, a paper was presented by J. Kemp under the title "Wave Guides in Electrical Communication"; this having been published prior to the meeting ( $J$. Inst. Elec. Eng., 90, Part 3, No. 11 ; September 1943). With the continuous extension of radio research and application to increasingly higher frequencies, the electrical engineer is already making considerable use of wave guides in place of the more conventional pair of wires or a co-axial cable. The paper under consideration comprises a most valuable survey of the state of published knowledge on this subject and a good introduction for those who have to become familiar with this new technique in the immediate futures.

The fundamental theoretical principles of the propagation of electric waves inside hollow conductors or 'guides' have been known for nearly half a century, as a result of the investigations of Sir J. J. Thomson, Sir Joseph Larmor and particularly of Lord Rayleigh, who, in 1897, first dealt with the sukject in a comprehensive manner, and established the foundations of the theory on which the modern structure rests. Although Lord Rayleigh's investigations were restricted to hollow metal tubes of infinite conductivity, of unlimited length and of arbitrary cross-section, he was able to show that two distinct classes of waves could be propagated down such a tube. These waves are distinguished by the fact that, in addition to the transverse electric and magnetic forces which accompany the transmission of waves along two parallel wires or a co-axial cable, there is a resultant longitudinal force, which is electric in one case $(E$ class wave) and magnetic in the other case ( $H$-class wave). Corresponding to the various modes of vibration of a circular membrane or of liquid in a cylindrical vessel, each of the above two classes of wave can be propagated in wave guides in various modes and orders, the essential features of which are described and illustrated in Mr. Kemp's paper.

Up to about 1930, the further investigation of the 'guiding' action of conductors on electric waves was largely confined to the study of transmission line technique or of the propagation of waves round the surface of the earth. Owing to the fact, shown by Lord Rayleigh, that wave-guide transmission could only take place when the wave-length was of the same order or less than the cross-sectional dimensions of the tube, the practical development of the subject had to await the availability of means for the production of very short waves. The paper under consideration shows that, during the past eight years or so, much of the experimental investigation and developrnent of wave-guide technique has been carried out in the United States, notably by G. C. Southworth, S. A. Schelkunoff, W. L. Barrow and their co-workers. All this and other work are summarized in an admirable manner in Mr. Kemp's paper, which describes and illustrates the way in which the various types of waves may be launched into guides, the attenuation and other conditions to which they are subject, and the means whereby they may be converted from one type to another, or may be amplified, detected and received for practical use. In addition, by providing suitably spaced apertures in a wave guide, or by terminating it in a flared horn of suitable size and shape, the wave energy propagated along the guide may be radiated into free space in the form of a concentrated or widespread beam analogous to the emission of longer waves from antenna arrays and reflector systems. Conversely, such horns may be used to receive incoming radiation and feed it into a suitable wave-guide on its way to the radio receiver.

As the author of the paper under consideration points out, the prospective field of application of wave guides embraces various systems of communication, providing telephone and television channels in numbers vastly exceeding those of any system of established type. When used in conjunction with horns, the guides may serve for broadcasting and television, for blind landing of aircraft, for detecting, locating and manceuvring of ships, and for other purposes such as radio telephone links, for which at present radiators and receivers of conventional type are used. The full extent of the field of application of wave guides will ultimately be governed by economic as weil as technical considerations, and must therefore remain largely a matter of conjecture; but, judged from the course the development has taken during recent years, it is likely that the field will be large and attractive.

\section{ARCH/EOLOGICAL EXCAVATION IN KASHMIR}

$\mathrm{D}^{\mathrm{H}}$ R. HELLMUT DE TERRA has issued an account of his investigations of the megaliths of Bursahom, Kashmir (Proc. Amer. Phil. Soc., 85, No. 5; September 1942). The valley of Kashmir has been, of course, of the greatest importance in Indian prehistory. It has been a channel through which cultures have penetrated southwards from west-central Asia on one hand and from the Tarim basin on the other. When the early Indus civilization, about which something is now known, thanks to the excavations at Mohenjo Daro, etc., began to wane, cultures from the far north gradually penetrated into northern India, and Kashmir was one of the routes taken by the immigrants. It is to be expected, then, that any early finds in Kashmir might well show foreign influences.

Bursahom is situated on a hilltop composed of pleistocene material, and near by is a megalithic monument recalling in shape a cromlech. Excavation revealed three layers. The uppermost yielded sherds belonging to the Buddhist period of the later IndoScythian dynasty of the fourth century. The second cultural level yielded hearths, loose stone foundations and five pits with urns containing the bones of pig and deer. Black burnished ware, decorated with incised geometric ornamentation, occurs. It is suggested that this culture shows influences from north-west or west-central Asia, and that the mega- 